\title{
DUALITY AND DIFFERENTIAL OPERATORS FOR HARMONIC MAASS FORMS
}

\author{
KATHRIN BRINGMANN, BEN KANE, AND ROBERT C. RHOADES \\ In memory of Leon Ehrenpreis
}

\section{Introduction And Statement of Results}

Fourier coefficients of automorphic forms play a prominent role in mathematics (see for instance [26]). Kloosterman sums arise naturally in the analytic theory of such coefficients. For instance, the Kuznetsov trace formula [29] relates a certain infinite sum related to the Fourier coefficients of automorphic forms to an infinite sum involving Kloosterman sums. The classical Poincaré series at infinity of weight $2<k \in \frac{1}{2} \mathbb{Z}$ on $\Gamma_{0}(N)$, denoted by $P(m, k, N ; z)$ (see (2.2) for the definition) with $m \in \mathbb{Z}, N \in \mathbb{N}$, and $z \in \mathbb{H}$, play an important role in such trace formulas.

The Poincaré series $P(m, k, N ; z)$ are elements of $M_{k}^{!}(N)$, the space of weakly holomorphic weight $k$ modular forms for $\Gamma_{0}(N)$, i.e., those meromorphic modular forms whose poles lie only at the cusps. Furthermore, if $m \geq 0$ then $P(m, k, N ; z)$ has bounded growth toward all cusps and so is in $M_{k}(N)$, the subspace of $M_{k}^{!}(N)$ of holomorphic modular forms. For $k>2$, $m \in \mathbb{Z}$ with $m<0$, and $n \in \mathbb{N}$, the $n$-th coefficient of $P(m, k, N ; z)$ equals (for example, see [23], Chapter 3)

$$
2 \pi i^{k}\left|\frac{n}{m}\right|^{\frac{k-1}{2}} \sum_{\substack{c>0 \\ c \equiv 0}} \frac{K_{k}(m, n, c)}{c} I_{k-1}\left(\frac{4 \pi \sqrt{|m n|}}{c}\right),
$$

where $I_{k-1}$ denotes the usual $I$-Bessel function and $K_{k}(m, n, c)$ denotes a certain Kloosterman sum (see (2.4) for the definition). For negative weights certain (possibly) non-holomorphic Poincaré series $F(m, 2-k, N ; z)$ are natural (see $(2.3)$ for the definition). Denote by $H_{w}(N)$ the space of harmonic Maass forms of weight $w$ on $\Gamma_{0}(N)$ (see Section 2 for the definition) and let $H_{w}^{\infty}(N)$ be the subspace of those elements of $H_{w}(N)$ that are bounded at all cusps other than $\infty$. The $n$-th Fourier coefficient of $F(m, 2-k, N ; z)$ is a sum involving Kloosterman sums $K_{2-k}(m, n, c)$ with a shape similar to (1.1). Series with Fourier expansions of this type play a prominent role in the works of Knopp, Rademacher, Zuckermann, and many others. See for instance [33].

Date: January 23, 2012.

2000 Mathematics Subject Classification. 11F37,11F25,11F30.

The first author was partially supported by NSF grant DMS-0757907 and the Alfried-Krupp prize. The third author is supported by an NSF Postdoctoral Fellowship and was supported by the Chair in Analytic Number Theory at EPFL during part of the preparation of this article. 
Due to the obvious symmetry $| \pm m n|=| \pm n m|$ and the simple relation $\left| \pm \frac{m}{n}\right|=\left| \pm \frac{n}{m}\right|^{-1}$, (1.1) reveals that several important results about coefficients of modular forms and harmonic Maass forms manifest themselves through the symmetries of the Kloosterman sum. Firstly, whenever $k \in \mathbb{Z}$ the Kloosterman sum is symmetric in $m$ and $n$. As a result, the $n$-th Fourier coefficient of $F(m, 2-k, N ; z)$ equals $\left|\frac{m}{n}\right|^{k-1}$ times the $m$-th Fourier coefficient of $F(n, 2-k, N ; z)$ (see [19], Theorem 3.4).

For $k \in \frac{1}{2} \mathbb{Z} \backslash \mathbb{Z}$ a slightly more complicated symmetry exists. Namely, (for a proof, see, for example, Proposition 3.1 of [8])

$$
K_{k}(m, n, c)=(-1)^{k+\frac{1}{2}} i K_{2-k}(n, m, c) .
$$

Consequentially, the $n$-th Fourier coefficient of $F(m, 2-k, N ; z)$ is essentially equal to the negative of the $m$-th Fourier coefficient of $P(n, k, N ; z)$. The resulting identities among Fourier coefficients are referred to as duality. Duality, in this context, was studied by Zagier [39], who showed that the traces of singular moduli are Fourier coefficients of a weight $\frac{1}{2}$ weakly holomorphic modular form and then related these traces to Fourier coefficients of weight $\frac{3}{2}$ modular forms. Zagier's work gave a new perspective on a result of Borcherds [5], relating what are now known as Borcherds products to coefficients of weakly holomorphic modular forms. To illustrate this famous result, consider the weight 4 Eisenstein series for $\mathrm{SL}_{2}(\mathbb{Z}),\left(q=e^{2 \pi i z}\right)$

$$
E_{4}(z):=1+240 \sum_{n \geq 1}\left(\sum_{d \mid n} d^{3}\right) q^{n}=(1-q)^{-240}\left(1-q^{2}\right)^{26760} \cdots=\prod_{n \geq 1}\left(1-q^{n}\right)^{c(n)} .
$$

Borcherds related the exponents $c(n)$ to the Fourier coefficients a certain weight $\frac{1}{2}$ weakly holomorphic modular form.

The proof through Kloosterman sums of the duality shown by Zagier outlined here is due to Jenkins [24]. This was later generalized by the first author and Ono [8] to a duality in the more general setting of harmonic Maass forms.

Duality has continued to be a central theme in the literature surrounding automorphic forms. For example, Bruinier and Ono [12] have shown a natural way to map the Borcherds exponents to coefficients of a $p$-adic modular form through a certain differential operator. Duality was extended by Folsom and Ono, and Zwegers [20,43] to relate coefficients of different mock modular forms. Duality has also been extended by Rouse [35] to Hilbert modular forms and to Maass-Jacobi forms by the first author and Richter [10].

For every $k \in \frac{1}{2} \mathbb{Z}$, a trival change of variables (namely $d \rightarrow-d$, see (2.4)) yields

$$
K_{2-k}(m, n, c)=\overline{K_{k}(-m,-n, c)},
$$

from which one obtains a natural relation between the $n$-th Fourier coefficient of $F(m, 2-k, N ; z)$ and the $-n$-th Fourier coefficient of $P(m, k, N ; z)$. This relation plays a prominent role in the theory of harmonic Maass forms. In particular, it governs the image of $F(m, 2-k, N ; z)$ under the weight $2-k$ antiholomorphic differential operator

$$
\xi_{2-k}:=2 i y^{2-k} \frac{\bar{\partial}}{\partial \bar{z}} .
$$


Since $\xi_{2-k}$ is essentially the Maass weight lowering operator (see (2.5) in Section 2.3), if $\mathcal{M} \in H_{2-k}^{\infty}(N)$, then $\xi_{2-k}(\mathcal{M})$ is a weight $k$ modular form. In particular, from (1.2) we may deduce that $\xi_{2-k}(F(m, 2-k, N ; z))$ equals a certain non-zero constant times $P(m, k, N ; z)$ (see $(2.8)$ for a precise statement). The surjectivity of $\xi_{2-k}$, first proven by Bruinier and Funke [11], follows.

Remark. We exclude the cases when the weight is $0 \leq k \leq 2$. In such cases, the convergence of the Poincaré series is delicate (see for example [30] and the expository survey [15]). Moreover, the Fourier expansions of modular forms of small weight are handled by Knopp [27] and for harmonic weak Maass forms of small weight by Pribitkin [31, 32].

1.1. Differential Operators via Kloosterman Sum Symmetries. We exploit another simple relation between Kloosterman sums. Whenever $k \in \mathbb{Z}$ there is an additional symmetry which occurs because the Kloosterman sum is independent of the weight $k \in \mathbb{Z}$. In particular,

$$
K_{k}(-m,-n, c)=K_{2-k}(-m,-n, c),
$$

so that (1.2) leads to a relation between the coefficients of $F(m, 2-k, N ; z)$ and $F(-m, 2-k, N ; z)$. We define the flipping operator $\mathcal{F}$ on Poincaré series by

$$
F(m, 2-k, N ; z) \mapsto F(-m, 2-k, N ; z) .
$$

Since $\{F(m, 2-k, N ; z): m \in \mathbb{Z}\}$ is a basis for $H_{2-k}^{\infty}(N)$, we may extend the operator $\mathcal{F}$ to all of $H_{2-k}^{\infty}(N)$ by linearity. Moreover, when $k>2$ and $\mathcal{M} \in H_{2-k}^{\infty}(N)$, the growth of $\mathcal{M}(z)$ as $z \rightarrow i \infty$ uniquely determines $\mathcal{M}$ as a linear combination of Poincaré series, and hence it is simple to determine the representation by this basis. Alternatively, for $f \in H_{2-k}^{\infty}(N)$ one may define $\mathcal{F}$ in terms of the weight raising operator by

$$
\mathcal{F}(f)=y^{k-2} \overline{R_{2-k}^{k-2}(f)},
$$

where $R_{2-k}^{k-2}$ is the $(k-2)$-fold Maass raising operator, as defined in (2.6). We investigate this connection in Section 2.3.

Remarks.

(1) After completion of this paper, the authors learned that the flipping operator is independently studied from a different perspective by Fricke and will be included in his forthcoming thesis [21] advised by Zagier. Moreover, the referee pointed out that the flipping operator appears in another context in the work of Knopp [25] and Knopp-Lehner [28].

(2) Denote by $M_{w}^{\infty}(N) \subseteq M_{w}^{!}(N)$ the subspace of those forms that are bounded at all cusps other than $\infty$. In this notation, the operator $\mathcal{F}$ gives a mapping

$$
\mathcal{F}=\mathcal{F}_{k, N}: H_{2-k}^{\infty}(N) \rightarrow H_{2-k}^{\infty}(N) / M_{2-k}(N)
$$

(3) Although we restrict ourselves in this paper to forms with bounded growth at cusps other than $\infty$, the general case would follow similarly after examining Poincaré series with growth only occurring in one of the other cusps. The cusp $\infty$ plays a prominent role here based on the fact that recent applications have emphasized forms with this property (see for example [8], [13], and [14]). 
The operator $D^{k-1}$, where $D:=\frac{1}{2 \pi i} \frac{\partial}{\partial z}$, serves as a counterpart to $\xi_{2-k}$ for $k \in 2 \mathbb{N}$. The role of $D^{k-1}$ in questions involving the algebraicity of Fourier coefficients is investigated in [14] and [22]. Here we exploit the symmetries given in (1.2) and (1.3) in order to relate the operators $D^{k-1}$ and $\xi_{2-k}$ through $\mathcal{F}$.

Theorem 1.1. For $k>2$ an integer and $\mathcal{M} \in H_{2-k}^{\infty}(N)$ we have

$$
D^{k-1}(\mathcal{M})=(-4 \pi)^{1-k} \Gamma(k-1) \xi_{2-k}(\mathcal{F}(\mathcal{M})) .
$$

Remark. If $\mathcal{M}(z)=\sum_{n \in \mathbb{Z}} c_{n}(y) e^{2 \pi i n x} \in H_{2-k}^{\infty}(N)$, then the operator $\xi_{2-k}$ may (essentially) be viewed as extracting those coefficients with $n<0$ while those with $n>0$ are extracted by $D^{k-1}$.

The above discussion suggests that we could proceed by directly calculating the Fourier expansions of Poincaré series. Computing the derivatives on the Fourier expansion and using the symmetries of the Kloosterman sums then yields the theorem. Instead we compute the derivatives on the Whittaker functions which are averaged to form the Poincaré series. This is possible because $D^{k-1}$ and $\xi_{2-k}$ are related to the Maass weight raising and lowering operators which commute with the action of $\Gamma_{0}(N)$. In fact, Bol's famous identity ([4], see also [18]) equates $D^{k-1}$ to the $(k-1)$-fold repeated application of the weight raising operator. The technique presented here does not directly use the symmetry given in (1.3) but rather works through the raising and lowering operators.

1.2. Applications of Flipping. We revisit some existing results and some results known to experts with the fresh perspective engendered by Theorem 1.1.

In Ramanujan's last letter to Hardy (see pages 127-131 of [34]), he introduced 17 examples of functions which he called mock theta functions. For example, he defined

$$
f(q):=1+\sum_{n=1}^{\infty} \frac{q^{n^{2}}}{\prod_{r=1}^{n}\left(1+q^{r}\right)^{2}} .
$$

He noted that they satisfied properties similar to modular forms (although he referred to modular forms as "theta functions") and also stated that certain linear combinations of his mock theta functions were indeed modular forms. Although many of these properties were proven over the course of the next 80 years (for example, see [1], [2], [3], [36], and [37]), however, even a rigorous definition of Ramanujan's mock theta functions failed to present itself. Zwegers [41, 42] finally placed Ramanujan's mock theta functions into a theoretical framework. In particular, if $h$ is a mock theta function, then he constructed an associated harmonic Maass form $\mathcal{M}_{h}$ such that the function

$$
g_{h}:=\xi_{\frac{1}{2}}\left(\mathcal{M}_{h}\right)=\xi_{\frac{1}{2}}\left(\mathcal{M}_{h}-h\right)
$$

is a unary theta function of weight 3/2. Following Zagier [40] we call $g_{h}$ the shadow of $h$.

By work of Bruinier-Funke [11], for any weakly holomorphic modular form $g$ of weight $k$ there exist a "mock-like" holomorphic function $h$ with shadow $g$. Following Zagier, we will call $h$ a mock modular form. More precisely, there is a harmonic Maass form $\mathcal{M}_{h}$ naturally associated to $h$ for which $\xi_{2-k}\left(\mathcal{M}_{h}\right)=\xi_{2-k}\left(\mathcal{M}_{h}-h\right)=g$. For each modular form $g$, we call the resulting harmonic Maass form a lift of $g$. 
The existence of a lift, or equivalently, the surjectivity of $\xi_{2-k}: H_{2-k}^{\infty}(N) \rightarrow M_{k}^{\infty}(N)$, combined with Theorem 1.1 implies that the operator $D^{k-1}$ is also surjective. Let $H_{w}^{\text {cusp }}(N)$ be the subspace of $H_{w}^{\infty}(N)$ that maps to $S_{2-w}(N)$, the subspace of weight $2-w$ cusp forms, under $\xi_{w}$. This gives the following theorem which is essentially contained in Theorems 1.1 and 1.2 of [14]. In [14], Nebentypus is allowed and the restriction that growth only occurs at the cusp $\infty$ is not made, but the image under $\xi_{w}$ is restricted to $S_{2-w}(N)$.

Theorem 1.2. If $2<k \in \mathbb{Z}$ and $\mathcal{M} \in H_{2-k}^{\infty}(N)$, then $D^{k-1}(\mathcal{M}) \in M_{k}^{\infty}(N)$. Moreover, in the notation of $(2.1)$,

$$
D^{k-1}(\mathcal{M}(z))=(-4 \pi)^{1-k}(k-1) ! c_{\mathcal{M}}^{-}(0)+\sum_{\substack{n \gg-\infty \\ n \neq 0}} c_{\mathcal{M}}^{+}(n) n^{k-1} q^{n} .
$$

The image of the map

$$
D^{k-1}: H_{2-k}^{\text {cusp }}(N) \longrightarrow M_{k}^{\infty}(N)
$$

consists of those $h \in M_{k}^{\infty}(N)$ which are orthogonal to cusp forms (see Section 3 for the definition) which also have constant term 0 at all cusps of $\Gamma_{0}(N)$. Furthermore, the map

$$
D^{k-1}: H_{2-k}^{\infty}(N) \longrightarrow M_{k}^{\infty}(N)
$$

is onto.

Implicit in the previous theorem are lifts of weakly holomorphic modular forms. Lifts of weight 3/2 unary theta functions were given by Zwegers [42]. He gave explicit constructions in terms of Lerch sums, yielding mock modular forms of weight $1 / 2$. Lifts of weight $1 / 2$ modular forms were constructed by the first author, Folsom, and Ono [6]. The forms they construct are related to the hypergeometric series occurring in the Rogers-Fine identity. Lifts of general cusp forms in $S_{k}(N)$ were treated by the first author and Ono in [9], using Poincaré series. Duke, Imamoḡlu, and Tóth [17] recently constructed lifts of the weight $\frac{3}{2}$ weakly holomorphic modular forms that are Zagier's traces of singular moduli generating functions [39].

The flipping operator extends the lift in [9] to a lift for all weakly holomorphic modular forms. Given $g(z)=\sum_{n \gg-\infty} c_{g}(n) q^{n} \in M_{k}^{\infty}(N)$ with $k>2$, define

$$
\mathcal{P}(g)(z):=(k-1)^{-1} \overline{c_{g}(0)} y^{k-1}-(4 \pi)^{1-k} \sum_{n \neq 0} \overline{c_{g}(-n)}|n|^{1-k} \Gamma(k-1 ;-4 \pi y n) q^{n},
$$

where $\Gamma(\alpha ; x):=\int_{x}^{\infty} e^{-t} t^{\alpha-1} d t$ is the incomplete gamma function. We note that for $g \in$ $S_{k}(N)$, our definition matches that of $4^{1-k} g^{*}$ given by Zagier [40]. The following theorem describes the lifts of interest, which will be given in terms of Poincaré series.

Theorem 1.3. For any $k \in \frac{1}{2} \mathbb{Z}, k>2, N \in \mathbb{N}$, and $g \in M_{k}^{\infty}(N)$, the following are true:

(1) There exists a harmonic Maass form $\mathcal{L}(g) \in H_{2-k}^{\infty}(N)$ such that

$$
\mathcal{L}(g)-\mathcal{P}(g)
$$

is a holomorphic function on $\mathbb{H}$.

(2) We have

$$
\xi_{2-k}(\mathcal{L}(g))=\xi_{2-k}(\mathcal{P}(g))=g
$$


Remark. The holomorphic function $\mathcal{L}(g)-\mathcal{P}(g)$ is typically not modular but mock modular. Theorem 1.3 allows us to deduce its transformation properties rather easily, since the transformation properies of $\mathcal{P}(g)$ may be deduced from the transformation properties of $g$.

The interrelation between weakly holomorphic modular forms and their lifts have led to better understanding of arithmetic information of both modular forms and harmonic Maass forms. The forms constructed by Duke, Imamoglu, and Tóth [17] are related to certain cycle integrals of modular functions. Bruinier, Ono, and the third author [14] showed that the vanishing of the Hecke eigenvalues of a Hecke eigenform $g$ implies the algebraicity of the coefficients of an appropriate lift of $g$. In other work, Bruinier and Ono [13] proved that the vanishing of the central value of the derivative of a weight 2 modular $L$-functions is related to the algebraicity of a certain Fourier coefficient of a harmonic Maass form.

Theorem 1.1 shows that for each $g \in S_{k}(N)$ one may find $M, M^{*} \in H_{2-k}^{\infty}(N)$ so that

$$
\xi_{2-k}(M)=g \quad \text { and } \quad D^{k-1}\left(M^{*}\right)=g .
$$

Recent work of Guerzhoy, Kent, and Ono [22] and the first two authors and Guerzhoy [7] shows that certain linear combinations of the these two "lifts" are $p$-adic modular forms. These works lead naturally to the following question: Let $M$ be a harmonic Maass form and set $g:=\xi_{2-k}(M)$ and $h:=D^{k-1}(M)$. From Theorem 1.3 we know that a harmonic Maass form $M^{*}$ exists such that $h=\xi_{2-k}\left(M^{*}\right)$. Is $g=D^{k-1}\left(M^{*}\right)$ ?

Corollary 1.4. Suppose that $k>2$ is an integer, $M \in H_{2-k}^{\infty}(N)$, and $g$ and $h$ are defined as above. If $M^{*} \in H_{2-k}^{\infty}(N)$ satisfies $\xi_{2-k}\left(M^{*}\right)=h$, then the projection of $D^{k-1}\left(M^{*}\right)$ onto the space of cusp forms is $\mathrm{g}$.

Furthermore, there exists a choice of $M^{*}$ such that $D^{k-1}\left(M^{*}\right)=g$.

Remark. In light of Theorems 1.1 and 1.2 , we may write $D^{k-1}\left(M^{*}\right)=g+\widetilde{g}$ with $\widetilde{g} \in$ $D^{k-1}\left(M_{2-k}^{\infty}(N)\right)$. The subspace $D^{k-1}\left(M_{2-k}^{\infty}(N)\right)$ has a number of exceptional properties. For example, the coefficients of a weakly holomorphic modular form in that space, when chosen to be algebraic, have high $p$-divisibility ([22], Proposition 2.1). Therefore it is natural to factor out by $M_{2-k}^{\infty}(N)$, and the statement of Corollary 1.4 may be taken to say that $M^{*} \equiv \mathcal{F}(M)\left(\bmod M_{2-k}^{\infty}(N)\right)$.

1.3. Choosing a Lift. As is suggested in Corollary 1.4, lifts are not unique because the kernel of $\xi_{2-k}$ is non-trivial. In fact, Bruinier and Funke [11] have shown that the kernel of $\xi_{2-k}$ is $M_{2-k}^{!}(N)$. The lift described in [9] is defined on Poincaré series and relations between the classical holomorphic Poincaré series make our lift unique up to a choice of a weakly holomorphic modular form. We present a procedure to make a choice of one such lift which is independent of the realization of $g \in M_{k}^{\infty}(N)$ as a linear combination of Poincaré series.

In order to describe the framework for our lift we will need to introduce some notation. For $M$ a harmonic Maass form with Fourier expansion as in (2.1), there is a polynomial $G_{M}(z)=\sum_{n \leq 0} c_{M}^{+}(n) q^{n} \in \mathbb{C}\left[q^{-1}\right]$ such that $M^{+}(z)-G_{M}(z)=O\left(e^{-\delta y}\right)$ as $y=\operatorname{Im}(z) \rightarrow \infty$ for some $\delta>0$. Here and throughout, we denote $z=x+i y$ with $x, y \in \mathbb{R}(y>0)$. We call $G_{M}$ the principal part of $M$ at infinity.

Let $M_{k}:=M_{k}(1)$ and define $H_{k}, S_{k}$, and $M_{k}^{!}$similarly. Given a weakly holomorphic form $g \in M_{k}^{!}$, we explicitly define a harmonic Maass form $G \in H_{2-k}$ such that $\xi_{2-k}(G)-g \in S_{k}$. 
Since the principal part of $g$ determines $g$ modulo forms in $S_{k}$, we will obtain a lift which is explicit and well defined if for every $g \in S_{k}$ we construct a unique, explicit lift $\widetilde{g} \in H_{2-k}$ with $\xi_{2-k}(\widetilde{g})-g=0$. The difficulty in this task lies in finding a lift which commutes with the algebra of $S_{k}$, so that $\widetilde{g}+\widetilde{h}=\widetilde{g+h}$ for $g, h \in S_{k}$. In particular, if one has two different bases for $S_{k}$, the lift must be independent of the basis representation. We call such a lift canonical.

Additionally, the lifts used in many applications are good choices of lifts (see Section 2 for the definition). We demonstrate a canonical lift for weakly holomorphic forms, which in the case of normalized Hecke eigenforms is good. To state our theorem, we introduce some notation. For $g \in S_{k}$ we denote the norm with respect to the usual Petersson scalar product by $\|g\|$. For $M \in H_{2-k}$, let

$$
A(M):=\inf \left\{n \in \mathbb{Z}: c_{M}^{+}(n) \neq 0\right\} .
$$

Theorem 1.5. Let $k>2$ and $g \in M_{k}^{!}$be given. Choose $M \in H_{2-k}$ with $A(M)$ maximal among all $M \in H_{2-k}$ with $\xi_{2-k}(M)=g$. Then $M$ is a canonical lift of $g$. Moreover, if $g \in S_{k}$ is a normalized Hecke eigenform, then $\|g\|^{-2} M$ is good for $g$.

Remark. For simplicity, we have constrained ourselves to the case of level 1 forms when considering canonical lifts. We will discuss the differences in the general level case briefly at the end of Section 4.

The paper is organized as follows: In Section 2 we recall some basic facts concerning harmonic Maass forms and Maass-Poincaré series and the relations between weight $2-k$ and weight $k$ Poincaré series given by the operators $\xi_{2-k}$ and $D^{k-1}$ (when $k \in \mathbb{N}$ ). In Section 3, we prove Theorems 1.1 and 1.2 as well as Theorem 1.3 and its corollaries. In Section 4, we prove Theorem 1.5.

\section{HARMONIC MAASS FORMS}

In this section we recall the definition of harmonic Maass form and the properties of harmonic Maass forms which are necessary to prove our results. A good reference for much of the theory recalled in this section is [11].

2.1. Basic notations and definitions. As usual it is assumed that if $k \in \frac{1}{2} \mathbb{Z} \backslash \mathbb{Z}$, then $N \equiv 0(\bmod 4)$. We define the weight $k$ hyperbolic Laplacian by

$$
\Delta_{k}:=-y^{2}\left(\frac{\partial^{2}}{\partial x^{2}}+\frac{\partial^{2}}{\partial y^{2}}\right)+i k y\left(\frac{\partial}{\partial x}+i \frac{\partial}{\partial y}\right)
$$

Moreover, for $\gamma=\left(\begin{array}{ll}a & b \\ c & d\end{array}\right) \in \mathrm{SL}_{2}(\mathbb{Z})$ when $k \in \mathbb{Z}$, respectively for $\gamma \in \Gamma_{0}(4)$ when $k \in \frac{1}{2} \mathbb{Z} \backslash \mathbb{Z}$ and any function $g: \mathbb{H} \rightarrow \mathbb{C}$, we let

$$
\left.g\right|_{k} \gamma(z):=j(\gamma, z)^{-2 k} g\left(\frac{a z+b}{c z+d}\right)
$$

where

$$
j(\gamma, z):= \begin{cases}\sqrt{c z+d} & \text { if } k \in \mathbb{Z}, \gamma \in \mathrm{SL}_{2}(\mathbb{Z}), \\ \left(\frac{c}{d}\right) \varepsilon_{d}^{-1} \sqrt{c z+d} & \text { if } k \in \frac{1}{2} \mathbb{Z} \backslash \mathbb{Z}, \gamma \in \Gamma_{0}(4),\end{cases}
$$


where for odd integers $d, \varepsilon_{d}$ is defined by

$$
\varepsilon_{d}:=\left\{\begin{array}{lll}
1 & \text { if } d \equiv 1 & (\bmod 4) \\
i & \text { if } d \equiv 3 & (\bmod 4)
\end{array}\right.
$$

Definition 2.1. A harmonic Maass form of weight $k$ on $\Gamma=\Gamma_{0}(N)$ is a smooth function $g: \mathbb{H} \rightarrow \mathbb{C}$ satisfying:

(i) $\left.g\right|_{k} \gamma(z)=g(z)$ for all $\gamma \in \Gamma$,

(ii) $\Delta_{k}(g)=0$,

(iii) $g$ has at most linear exponential growth at each cusp of $\Gamma$.

We note that $\mathcal{M} \in H_{w}(N)$ for $w \leq \frac{1}{2}$ has a Fourier expansion of the shape

$$
\mathcal{M}(z)=c_{\mathcal{M}}^{-}(0) y^{1-w}+\sum_{\substack{n \ll+\infty \\ n \neq 0}} c_{\mathcal{M}}^{-}(n) \Gamma(1-w ;-4 \pi n y) q^{n}+\sum_{n \gg-\infty} c_{\mathcal{M}}^{+}(n) q^{n}
$$

We call $\mathcal{M}^{+}(z):=\sum_{n \gg-\infty} c_{\mathcal{M}}^{+}(n) q^{n}$ the holomorphic part of $\mathcal{M}$ and $\mathcal{M}^{-}:=\mathcal{M}-\mathcal{M}^{+}$the non-holomorphic part of $\mathcal{M}$.

Following [14], one says that a harmonic Maass form $f \in H_{2-k}(N)$ is good for a normalized Hecke eigenform $g \in S_{k}(N)$ if it satisfies the following properties:

(1) The principal part of $f$ at the cusp $\infty$ belongs to $F_{g}\left[q^{-1}\right]$, with $F_{g}$ the number field obtained by adjoining the coefficients of $g$ to $\mathbb{Q}$.

(2) The principal parts of $f$ at the other cusps of $\Gamma_{0}(N)$, defined analogously, are constant.

(3) We have $\xi_{2-k}(f)=\|g\|^{-2} g$.

One sees immediately by the second condition that $f \in H_{2-k}^{\infty}(N)$.

2.2. Poincaré series. We describe two families of Poincaré series. Let $m$ be an integer, and let $\varphi_{m}: \mathbb{R}^{+} \rightarrow \mathbb{C}$ be a function which satisfies $\varphi_{m}(y)=O\left(y^{\alpha}\right)$, as $y \rightarrow 0$, for some $\alpha \in \mathbb{R}$. With $e(r):=e^{2 \pi i r}$, let

$$
\varphi_{m}^{*}(z):=\varphi_{m}(y) e(m x)
$$

Such functions are fixed by the translations, elements of $\Gamma_{\infty}:=\left\{ \pm\left(\begin{array}{ll}1 & n \\ 0 & 1\end{array}\right): n \in \mathbb{Z}\right\}$. Given this data define the generic Poincaré series

$$
\mathbb{P}\left(m, k, \varphi_{m}, N ; z\right):=\left.\sum_{A \in \Gamma_{\infty} \backslash \Gamma_{0}(N)} \varphi_{m}^{*}\right|_{k} A(z) .
$$

We note that the Poincaré series $\mathbb{P}\left(m, k, \varphi_{m}, N ; z\right)$ converges absolutely for $k>2-2 \alpha$, where $\alpha$ is the growth factor of $\varphi_{m}(y)$ as given above and by construction satisfies the modularity property $\left.\mathbb{P}\left(m, k, \varphi_{m}, N ; z\right)\right|_{k} \gamma(z)=\mathbb{P}\left(m, k, \varphi_{m}, N ; z\right)$ for every $\gamma \in \Gamma_{0}(N)$. In this notation, the classical family of holomorphic Poincaré series (see for example [23], Chapter 3) for $k \geq 2$ is given by

$$
P(m, k, N ; z)=\mathbb{P}(m, k, e(i m y), N ; z) .
$$

The Maass-Poincaré series (see for example [19]) are defined by

$$
F(m, 2-k, N ; z):=\mathbb{P}\left(-m, 2-k, \varphi_{-m}, N ; z\right),
$$


where

$$
\varphi_{-m}(z):= \begin{cases}\mathcal{M}_{1-\frac{k}{2}}(-4 \pi m y) & \text { if } k<0 \text { and } m \neq 0, \\ |m|^{1-k} \mathcal{M}_{\frac{k}{2}}(-4 \pi m y) & \text { if } k>2 \text { and } m \neq 0, \\ 1 & \text { if } k<0, m=0, \\ (4 \pi y)^{k-1} & \text { if } k>2, m=0 .\end{cases}
$$

Here for complex $s$

$$
\mathcal{M}_{s}(y):=|y|^{\frac{k}{2}-1} M_{\left(1-\frac{k}{2}\right) \operatorname{sgn}(y), s-\frac{1}{2}}(|y|),
$$

where $M_{\nu, \mu}(z)$ is the usual $M$-Whittaker function.

Since $\varphi_{m}^{*}$ is annihilated by the hyperbolic Laplacian and $\Delta_{2-k}$ commutes with the weight $2-k$ group action of $\Gamma_{0}(N)$, a consideration of the growth of $\varphi_{m}^{*}$ at all of the cusps shows that $F(m, 2-k, N ; z) \in H_{2-k}^{\infty}(N)$. In the case $k<0$ one has

$$
F(m, 2-k, N ; z)=P(-m, 2-k, N ; z) .
$$

In order to describe the coefficients of the Poincaré series we define the Kloosterman sums

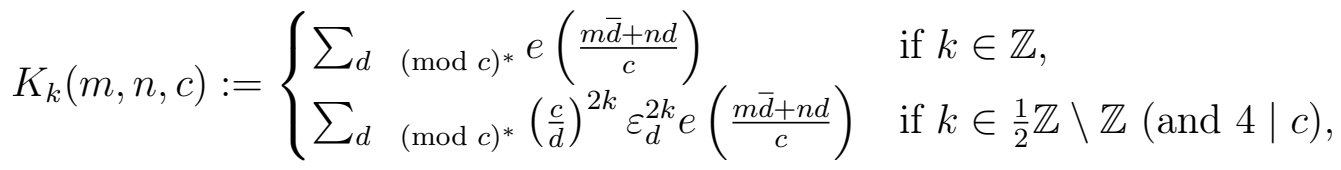

where $\left(\frac{c}{d}\right)$ denotes the Jacobi symbol. Here $d$ runs through the primitive residue classes modulo $c$, and $\bar{d}$ is defined by the congruence $d \bar{d} \equiv 1(\bmod c)$.

A calculation analogous to that for Theorem 3.4 of [19] yields the following result.

Lemma 2.2. If $k>2$ and $m \in \mathbb{Z}$ then the principal part of $F(m, 2-k, N ; z)$ is

$$
\delta_{m>0} \Gamma(k)|m|^{1-k} q^{-m}+c(m, k, N),
$$

where $\delta_{m>0}=1$ if $m>0$ and 0 otherwise, and $c(m, k, N)$ is a constant depending on $k, m$, and $N$. When $k \in 2 \mathbb{Z}$, we have

$$
c(m, k, N)=-(2 \pi i)^{k} \sum_{c \equiv 0} \frac{K_{2-k}(-m, 0, c)}{c^{k}} .
$$

The principal part of $P(m, k, N ; z)$ is $\delta_{m \leq 0} q^{m}$.

Remark. For $m \in \mathbb{Z}$ and $k \in 2 \mathbb{Z}$ we have $c(m, k, N)=(-1)^{k} \overline{c(-m, k, N)}$.

Moreover, the full Fourier expansion of $F(m, k, N ; z)$ is computed in Theorem 3.4 of [19]. We omit the full Fourier expansion, however, because it is not needed for our purposes.

2.3. Raising and Lowering Operators. The Maass raising and lowering operators are given by

$$
R_{k}:=2 i \frac{\partial}{\partial z}+k y^{-1} \text { and } L_{k}:=-2 i y^{2} \frac{\partial}{\partial \bar{z}} .
$$

For a real analytic function $f$ satisfying the weight $k$ modularity property $\left.f\right|_{k} \gamma(z)=f(z)$ for every $\gamma \in \Gamma_{0}(N)$ which is an eigenfunction under $\Delta_{k}$ with eigenvalue $s, R_{k}(f)(z)$ (respectively 
$\left.L_{k}(f)(z)\right)$ satisfies weight $k+2$ (resp. $k-2$ ) modularity and is an eigenfunctions under $\Delta_{k+2}$ (resp. $\Delta_{k-2}$ ) with eigenvalue $s+k$ (resp. $s-k+2$ ). This follows by the commutator relation

$$
-\Delta_{k}=L_{k+2} R_{k}+k=R_{k-2} L_{k} .
$$

Define for a positive integer $n$

$$
R_{k}^{n}:=R_{k+2(n-1)} \circ \cdots R_{k+2} \circ R_{k}
$$

and let $R_{k}^{0}$ be the identity. If $f \in H_{2-k}^{\infty}(N)$, then $f^{*}:=y^{k-2} \overline{R_{2-k}^{k-2}(f)} \in H_{2-k}^{\infty}(N)$, as noted in Remark 7 in [14]. Furthermore, by Bol's identity ([4], see also [18]), that is

$$
R_{2-k}^{k-1}=(-4 \pi)^{k-1} D^{k-1},
$$

one has (for $f \in H_{2-k}^{\text {cusp }}(N)$ see Remark 7 in [14]) that

$$
\xi_{2-k}\left(f^{*}\right)=y^{-k} \overline{L_{k}\left(f^{*}\right)}=R_{2-k}^{k-1}(f)=(-4 \pi)^{k-1} D^{k-1}(f) .
$$

So, up to a constant factor, $M^{*}$ behaves as $\mathcal{F}(f)$ under $\xi_{2-k}$. On the other hand one may compute the Fourier expansion of $f^{*}$ and see that it is the same as that for $\mathcal{F}(f)$. In this paper, we proceed differently and come about $\mathcal{F}$ on the level of Poincaré series.

2.4. Derivatives of Poincaré Series. The following relations, derived in the lemma below, are important for deducing the theorems of this paper.

Lemma 2.3. For $m \in \mathbb{Z}$, the action of the operators $\xi_{2-k}$ and $D^{k-1}$ on $F(m, 2-k, N ; z)$ is given by

$$
\begin{aligned}
& \xi_{2-k}(F(m, 2-k, N ; z))=(k-1)(4 \pi)^{k-1} P(m, k, N ; z), \\
& D^{k-1}(F(m, 2-k, N ; z))=\Gamma(k)(-1)^{k-1} P(-m, k, N ; z),
\end{aligned}
$$

where in (2.9) we require $k$ to be an integer.

Proof. For $m>0$, the relation (2.8) is noted (up to the constant) in Remark 3.10 of [11], while the constant is explicitly computed in Theorem 1.2 of [9]. The $m>0$ case of (2.9) is given in (6.8) of [14].

The lemma follows from the following relations. For $k>2$ we have

$$
\xi_{2-k}\left(\varphi_{m}^{*}\right)=(k-1)(4 \pi)^{k-1} q^{m} .
$$

Additionally, whenever $k$ is an even integer, we have

$$
D^{k-1}\left(\varphi_{m}^{*}\right)=-\Gamma(k) q^{-m} .
$$

The relations (2.10) and (2.11) together with the fact that $\xi_{2-k}$ and $D^{k-1}$ commute with the group law will immediately imply (2.8) and (2.9). Since the six calculations $(m<0, m=0$, and $m>0$ for each) to establish (2.10) and (2.11) are all similar, we include only the case of $D^{k-1}\left(\varphi_{-m}(z)\right)$ with $m<0$. In this case, we have

$$
\varphi_{-m}^{*}(z)=|m|^{1-k} e^{-2 \pi i m x}(4 \pi|m| y)^{\frac{k}{2}-1} M_{1-\frac{k}{2}, \frac{k-1}{2}}(4 \pi|m| y) .
$$


Applying the change of variables $2 \pi|m| y \rightarrow y$ and $2 \pi|m| x \rightarrow x$ and relations between the $W$-Whittaker and $M$-Whittaker functions (see page 346 of [38]), we consider

$$
|m|^{1-k} e^{i x}(2 y)^{\frac{k}{2}-1}\left((k-1) \exp \left(\pi i\left(1-\frac{k}{2}\right)\right) W_{\frac{k}{2}-1, \frac{k-1}{2}}(-2 y)-(-1)^{k} \Gamma(k) W_{1-\frac{k}{2}, \frac{k-1}{2}}(2 y)\right),
$$

for which we denote the two terms as $f_{1}(z)+f_{2}(z)$. Direct computation gives

$$
\frac{\partial}{\partial \bar{z}}\left(f_{2}\right)(z)=0 \quad \text { and } \quad \frac{\partial}{\partial x}\left(f_{2}\right)(z)=i f_{2}(z) \text {. }
$$

Hence, using $\frac{\partial}{\partial z}=\frac{\partial}{\partial x}-\frac{\partial}{\partial \bar{z}}$ we obtain

$$
D^{k-1}\left(f_{2}\right)(2 \pi z)=f_{2}(2 \pi z) .
$$

Thus a change of variables and using $W_{1-\frac{k}{2}, \frac{k}{2}-1}(2 y)=(2 y)^{1-\frac{k}{2}} e^{-y}$ yields

$$
D^{k-1}\left(f_{2}\right)(2 \pi|m| z)=(-1)^{k-1} \Gamma(k) q^{-m} .
$$

It remains to show that $D^{k-1}\left(f_{1}\right)(z)=0$. For this, let

$$
g_{r}(z):=|m|^{1-k} e^{i x}(-2 y)^{\frac{k}{2}-r} W_{\frac{k}{2}-r, \frac{k-1}{2}}(-2 y) .
$$

From the third three term recurrence relation

$$
y W_{k, m}^{\prime}(y)=\left(k-\frac{y}{2}\right) W_{k, m}(y)-\left(m^{2}-\left(k-\frac{1}{2}\right)^{2}\right) W_{k-1, m}(y)
$$

for the Whittaker function (see pages 350-352 of [38]) giving a relation for the derivative of the $W$-Whittaker function, we obtain

$$
\frac{\partial}{\partial z} g_{r}(z)=-\frac{i}{2 y}(k-2 r) g_{r}(z)+i\left(r^{2}-r(k-1)\right) g_{r+1}(z)
$$

Hence,

$$
R_{2 r-k}\left(g_{r}\right)(2 \pi z)=\left(r^{2}-r(k-1)\right) g_{r+1}(2 \pi z) .
$$

Using this for $r=k-1$ and applying Bol's identity (2.7), we have

$$
D^{k-1}\left(f_{1}\right)(2 \pi z)=(k-1) R_{2-k}^{k-1}\left(g_{1}\right)(2 \pi z)=0
$$

as desired.

2.5. Bol's Identity. Bol's identity (2.7) states that $D^{k-1}$ is essentially (up to a non-zero constant multiple) equal to $R_{2-k}^{k-1}$. The calculations of the previous section give the action of $R_{2-k}^{k-1}$ on the Whittaker functions which define the Poincaré series that span the spaces of forms of interest in this paper and then we use the commutation relation

$$
\left.\left(R_{2-k}^{k-1}(f)\right)\right|_{k} \gamma(z)=R_{2-k}^{k-1}\left(\left.f\right|_{2-k} \gamma\right)(z)
$$

between $R_{2-k}^{k-1}$ and the slash operator, valid for every real analytic function $f$. Alternatively, we can proceed by computing the Fourier expansion of the Maass-Poincaré series, obtaining an expansion as in (2.1). Differentiating term by term yields equations (2.8) and (2.9). This 
approach does not rely on the fact that the differential operator $D^{k-1}$ commutes with the group action (which would follow from Bol's identity). Additionally, for integral $k$ we have

$$
q^{m} \Gamma(k-1 ;-4 \pi m y)=\bar{q}^{m} Q_{k, m}(y)
$$

where $Q_{k, m}$ is a polynomial of degree at most $k-2$. Thus a direct computation of $D^{k-1}$ avoids an application of Bol's identity.

\section{Proof of Theorems 1.3, 1.1, And 1.2 And Corollary 1.4}

Having established the necessary preliminaries, we are now ready to prove Theorem 1.3.

Proof of Theorem 1.3. Since the Poincaré series $\{F(m, k, N ; z)\}_{m \in \mathbb{Z}}$ span $M_{k}^{\infty}(N)$ and the series $\{F(m, 2-k, N ; z)\}_{m \in \mathbb{Z}}$ span $H_{2-k}^{\infty}(N)$ it is enough to prove the result on the level of Poincaré series. Part (1) follows from equation (2.8) together with (2.1) and the fact that

$$
\xi_{2-k}(\mathcal{P}(P(m, k, N ; z)))=P(m, k, N ; z) .
$$

In particular,

$$
\frac{(4 \pi)^{1-k}}{k-1} F(m, 2-k, N ; z)-\mathcal{P}(P(m, k, N ; z))
$$

is the desired holomorphic function associated to the modular form $P(m, k, N ; z)$. Part (2) follows from (2.8).

Having established the image of the Poincaré series under the operators $D^{k-1}$ and $\xi_{2-k}$ in Section 2.4, the fact that the Poincaré series form a basis will suffice to prove Theorem 1.1.

Proof of Theorem 1.1. The proof of this result follows immediately from (2.8) and (2.9).

Borcherds [5] has defined a regularized inner product $(g, h)^{r e g}$ for $g, h \in M_{k}^{\infty}(N)$ from which one can define orthogonality in the more general setting of weakly holomorphic modular forms. For cusp forms $g$ and $h$, the regularized inner product reduces to the classical Petersson inner product. For $M \in H_{2-k}^{\text {cusp }}(N)$, we define

$$
h:=\Gamma(k-1) \mathcal{F}(M) \in H_{2-k}^{\infty}(N) .
$$

By Lemma 2.2 and the remark following it, the constant terms of $h$ and $M$ satisfy

$$
c_{h}^{+}(0)=\Gamma(k-1)(-1)^{k} \overline{c_{M}^{+}(0)}
$$

Combining this with Theorem 4.1 of [14] immediately leads to the following lemma (with the factor $\Gamma(k-1)$ correcting a typo from the original statement of Theorem 4.1), which is the most important computation toward calculating the image of $D^{k-1}$.

Lemma 3.1. If $g \in M_{k}(N)$ and $M \in H_{2-k}^{\text {cusp }}(N)$, then

$$
(-4 \pi)^{k-1}\left(g, D^{k-1}(M)\right)^{r e g}=\frac{(-1)^{k} \Gamma(k-1)}{\left[\mathrm{SL}_{2}(\mathbb{Z}): \Gamma_{0}(\mathrm{~N})\right]} \sum_{\kappa \in \Gamma_{0}(N) \backslash P^{1}(\mathbb{Q})} w_{\kappa} \cdot c_{g}(0, \kappa) \overline{c_{M}^{+}(0, \kappa)},
$$

where $c_{g}(0, \kappa)$ (resp. $\left.c_{M}^{+}(0, \kappa)\right)$ denotes the constant term of the Fourier expansion of $g$ (resp. $M)$ at the cusp $\kappa \in P^{1}(\mathbb{Q})$, and $w_{\kappa}$ is the width of the cusp $\kappa$. 
Proof of Theorem 1.2. The first part of the theorem follows from Theorem 1.1 and (2.9). The surjectivity of $D^{k-1}$ on $H_{2-k}^{\infty}(N)$ follows from the surjectivity of $\xi_{2-k}$ (see Theorem 3.7 of [11]) and Theorem 1.1.

Additionally, if $\mathcal{M} \in H_{2-k}^{\text {cusp }}(N)$, it follows from the first part of the theorem and (2.8) that there exist $\alpha_{m} \in \mathbb{C}$ so that

$$
\mathcal{M}(z)=\sum_{m>0} \alpha_{m} F(m, 2-k, N ; z)
$$

Thus from Lemma 3.1 and the first part of the theorem, $D^{k-1}(\mathcal{M})$ is orthogonal to cusp forms and the constant term at each cusp of $\Gamma_{0}(N)$ vanishes.

Conversely, assume that $h \in M_{k}^{\infty}(N)$ has vanishing constant term at any cusp of $\Gamma_{0}(N)$ and is orthogonal to cusp forms. From (2.9) we may take

$$
f(z)=\sum_{m \in \mathbb{N}} \alpha_{m} F(m, 2-k, N ; z) \in H_{2-k}^{\text {cusp }}(N)
$$

such that the principal parts of $D^{k-1}(f)$ and $h$ at the cusps agree. Consequently, $h-$ $D^{k-1}(f) \in S_{k}(N)$. In view of Lemma 3.1, the hypothesis on $h$ and (2.9), we find that $h-D^{k-1}(f)$ is orthogonal to cusp forms. Hence it vanishes identically.

We conclude with the proof of Corollary 1.4.

Proof of Corollary 1.4. Writing $M \in H_{2-k}^{\infty}(N)$ in terms of Poincaré series we have

$$
M(z)=\sum_{m \in \mathbb{Z}} \alpha_{m} F(m, 2-k, N ; z) .
$$

Then Theorem 1.1 implies that $(-4 \pi)^{1-k} \Gamma(k-1) \mathcal{F}(M)$ is a lift of $h=D^{k-1}(M)$ and

$$
M^{*}-(-4 \pi)^{1-k} \Gamma(k-1) \mathcal{F}(M) \in M_{2-k}^{\infty}(N),
$$

where $M^{*} \in H_{2-k}^{\infty}(N)$ is any harmonic Maass form satisfying $\xi_{2-k}\left(M^{*}\right)$, as given in the statement of Corollary 1.4. Applying Theorems 1.1 and 1.2 we obtain the assertion concerning $D^{k-1}\left(M^{*}\right)$.

\section{A CANONICAL LIFT}

When $N=1$ we use the abbreviations $P(m, k ; z):=P(m, k, 1 ; z)$ and $F(m, k ; z):=$ $F(m, k, 1 ; z)$. For fixed $k>2$ integral, let $\ell:=\operatorname{dim} S_{k}$ and define $f_{2-k, m} \in M_{2-k}^{!}$to be the unique weakly holomorphic modular form satisfying

$$
f_{2-k, m}(z)=q^{-m}+O\left(q^{-\ell}\right) .
$$

Such weakly holomorphic modular forms were explicitly constructed in [16] as

$$
f_{2-k, m}(z):=\left\{\begin{array}{cl}
E_{k^{\prime}}(z) \Delta(z)^{-\ell-1} F_{m}(j(z)) & \text { if } m>\ell, \\
0 & \text { if } m \leq \ell .
\end{array}\right.
$$

Here $k^{\prime} \in\{0,4,6,8,10,14\}$ with $k^{\prime} \equiv 2-k(\bmod 12), E_{k^{\prime}}$ is the Eisenstein series of weight $k^{\prime}, \Delta$ is the unique normalized Hecke eigenform of weight 12 , and $F_{m}$ is a generalized Faber 
polynomial of degree $m-\ell-1$ constructed recursively in terms of $f_{2-k, m^{\prime}}$ with $m^{\prime}<m$ to cancel higher powers of $q$. Finally, for $m \in \mathbb{Z}$ define

$$
G_{m, 2-k}(z):=F(m, 2-k ; z)-\delta_{m>0} \Gamma(k)|m|^{1-k} f_{2-k, m}(z) .
$$

Here $\delta_{m>0}$ is defined as in Lemma 2.2. From Lemma 2.2 and the definition of $f_{2-k, m}$, the holomorphic part $G_{m, 2-k}^{+}(z)$ of $G_{m, 2-k}(z)$ satisfies

$$
G_{m, 2-k}^{+}(z)=O\left(q^{-\ell}\right) .
$$

The following explicit theorem implies Theorem 1.5.

Theorem 4.1. Suppose that $2<k \in 2 \mathbb{Z}$ and $g \in M_{k}^{!}$and write $g(z)=\sum_{m \in I} a_{m} P(m, k ; z)$ for some index set $I \subset \mathbb{Z}$. Then the $\xi_{2-k}$-preimage choice

$$
\mathcal{L}(g(z))=\mathcal{L}_{I}(g(z)):=\frac{1}{k-1} \sum_{m \in I} \frac{\overline{a_{m}}}{(4 \pi)^{k-1}} G_{m, 2-k}(z)
$$

defines a canonical lifting from $M_{k}^{!}$to $H_{2-k}$. Moreover, when $g \in S_{k}$ is a normalized Hecke eigenform, the lift $\mathcal{L}(\mathrm{g}) /\|g\|^{2}$ is good for $g$.

Proof. One directly obtains that $\xi_{2-k}(\mathcal{L}(g(z)))=g$ from (2.8). Consider

$$
\mathscr{G}(z):=\sum_{\substack{m \in I \\ m \leq 0}} a_{m} P(m, k ; z),
$$

Then $g-\mathscr{G} \in S_{k}$. Set

$$
\mathcal{H}(z):=\mathcal{L}(\mathscr{G}(z))=\frac{1}{k-1} \sum_{n \leq 0} \frac{\overline{a_{n}}}{(4 \pi)^{k-1}} F(n, 2-k ; z) .
$$

The following lemma, which is proved after we conclude the proof of Theorem 4.1, shows that $\mathcal{H} \in H_{2-k}$ is the unique lift of $\mathscr{G}$ with $\mathcal{H}^{+}$having minimal growth at the cusp $\infty$.

Lemma 4.2. With $g$ as in Theorem 4.1, the function $\mathcal{H}$ is the unique $h \in H_{2-k}$ whose holomorphic part exhibits sub-exponential growth at the cusp $\infty$ and satisfies

$$
g-\xi_{2-k}(h) \in S_{k} .
$$

Applying Lemma 4.2 we may assume that $g$ is a cusp form in order to prove Theorem 4.1. We write $g(z)=\sum_{m \in I} a_{m} P(m, k ; z)$ with some index set $I \subset \mathbb{N}$. From (4.2) we obtain

$$
\mathcal{L}_{I}(g(z))=\frac{1}{k-1} \sum_{m \in I} \frac{\overline{a_{m}}}{(4 \pi)^{k-1}} G_{m, 2-k}(z)=O\left(q^{-\ell}\right) .
$$

To show that the lift is independent of the choice of the index set, let $J \subset \mathbb{N}$ be given such that $g(z)=\sum_{m^{\prime} \in J} a_{m^{\prime}} P\left(m^{\prime}, k ; z\right)$. Then

$$
\mathcal{L}_{I}(g(z))-\mathcal{L}_{J}(g(z))=O\left(q^{-\ell}\right)
$$

and

$$
\xi_{2-k}\left(\mathcal{L}_{I}(g)-\mathcal{L}_{J}(g)\right)=g-g=0
$$


Hence

$$
\mathcal{L}_{I}(g)-\mathcal{L}_{J}(g) \in \operatorname{ker}\left(\xi_{2-k}\right)=M_{2-k}^{!} .
$$

By the valence formula, we know that a weakly holomorphic modular form $h$ that satisfies $h(z)=O\left(q^{-\ell}\right)$ must be 0 . Therefore combining equations (4.4) and (4.5) yields

$$
\mathcal{L}_{I}(g)=\mathcal{L}_{J}(g)
$$

This finishes the proof of the first statement of the theorem.

To prove the second statement, assume that $g \in S_{k}$ is a normalized Hecke eigenform and $h \in H_{2-k}$ is a harmonic Maass form which is good for $g$. Thus the principal part of $h$ is $\sum_{n \leq 0} c_{n} q^{n}$ with $c_{n} \in K_{g}$. By comparing principal parts, we have

$$
h(z)=\sum_{n>0} \frac{c_{-n} n^{k-1}}{\Gamma(k)} F(n, 2-k ; z),
$$

since the difference has bounded principal part and maps to a cusp form under $\xi_{2-k}$. We have

$$
\xi_{2-k}(h)(z)=\sum_{n>0} \frac{\overline{c_{-n}}}{\Gamma(k)}(k-1)(4 \pi n)^{k-1} P(n, k ; z)=\frac{g(z)}{\|g\|^{2}} .
$$

Set $I:=\left\{n \leq 0: c_{n} \neq 0\right\}$. By definition

$$
\mathcal{L}\left(\frac{g}{\|g\|^{2}}\right)(z)=\mathcal{L}_{I}\left(\frac{g}{\|g\|^{2}}\right)(z)=\sum_{n>0} \frac{c_{-n} n^{k-1}}{\Gamma(k)} F(n, 2-k ; z)-\sum_{n>0} c_{-n} f_{2-k, n}(z) .
$$

It follows that

$$
\left(h-\mathcal{L}\left(\frac{g}{\|g\|^{2}}\right)\right)=\sum_{n>0} c_{-n} f_{2-k, n} .
$$

Since $E_{k^{\prime}}, \Delta^{-1}$, and $F_{m}(j(\tau))$ all have rational (furthermore, integer) coefficients, the weakly holomorphic modular forms $f_{2-k, n}$ have rational coefficients by equation (4.1). It follows that $h-\mathcal{L}\left(g /\|g\|^{2}\right)$ has coefficients in $K_{g}$. Therefore, since the coefficients of the principal part of $h$ and the principal part of $h-\mathcal{L}\left(g /\|g\|^{2}\right)$ are both in $K_{g}$, it follows that the coefficients of the principal part of $\mathcal{L}\left(g /\|g\|^{2}\right)$ are contained in $K_{g}$. Hence $\mathcal{L}\left(g /\|g\|^{2}\right)$ is also a good lift for $g$.

Proof of Lemma 4.2. Using (2.8) together with the fact that $P(m, k ; z) \in S_{k}$ for $m \geq 1$ immediately implies (4.3). To show uniqueness, let $h \in H_{2-k}$ satisfy (4.3). Since the Poincaré series $P(n, k ; z)$ span the space $M_{k}^{!}$, it follows that

$$
\xi_{2-k}(h(z))=\sum_{n \in \mathbb{Z}} b_{n} P(n, k ; z)
$$

for some $b_{n} \in \mathbb{C}$. By equation (4.3) we have that

$$
g(z)-\sum_{n \in \mathbb{Z}} b_{n} P(n, k ; z) \in S_{k} .
$$


Comparing the principal parts of both summands in (4.6), one sees that $b_{n}=a_{n}$ for every $n \leq 0$. It follows that

$$
h(z)-\mathcal{H}(z)=\frac{(4 \pi)^{1-k}}{k-1} \sum_{n>0} \overline{b_{n}} F(n, 2-k ; z) .
$$

This has principal part (up to the constant term) equal to

$$
\Gamma(k-1) \sum_{n>0}(4 \pi n)^{1-k} \overline{b_{n}} q^{-n}
$$

and hence exhibits exponential growth at $\infty$ unless $b_{n}=0$ for every $n>0$. This establishes the uniqueness of $\mathcal{H}$.

Remark. We now briefly discuss the canonical lift for non-trivial level. For $\mathscr{G}$ such that $g-\mathscr{G} \in S_{k}(N)$ one merely defines $\mathcal{L}(\mathscr{G})$ by replacing $F(n, 2-k ; z)$ with $F(n, 2-k, N ; z)$. In order to obtain a lift for $g \in S_{k}(N)$, we choose $m_{N}>0$ to be minimal such that there exists $j_{N}^{*} \in M_{0}^{\infty}(N)$ with $j_{N}^{*}(z)=q^{-m_{N}}+O\left(q^{-\left(m_{N}-1\right)}\right)$. The condition that (1.4) is maximal among all lifts $M$ of a form $g \in S_{k}$ will be further refined to the condition that

$$
A(M, r):=\inf \left\{n \in \mathbb{Z}: n \equiv r \quad\left(\bmod m_{N}\right), c_{M}^{+}(n) \neq 0\right\}
$$

is maximal for every $r \in\left\{0, \ldots, m_{N}-1\right\}$.

\section{REFERENCES}

[1] G. Andrews, On the theorems of Watson and Dragonette for Ramanujan's Mock theta functions, Amer. J. Math. 88 (1966), 454-490.

[2] G. Andrews, The fifth and seventh order mock theta functions, Trans. Amer. Math Soc. 293 (1986), $113-134$.

[3] G. Andrews and D. Hickerson, Ramanujan's lost notebook VII. The sixth order mock theta functions, Adv. Math. 89, (1991), 60-105.

[4] G. Bol, Invarianten linearer Differentialgleichungen, Abh. Mat. Sem. Univ. Hamburg 16 (1949), 1-28.

[5] R. Borcherds, Automorphic forms with singularities on Grassmannians, Invent. Math. 132 (1998), 491562.

[6] K. Bringmann, A. Folsom, and K. Ono, q-series and weight 3/2 Maass forms, Compositio Math. 145 (2009), 541-552.

[7] K. Bringmann, P. Guerzhoy, and B. Kane, Mock Modular Forms as p-adic Modular Forms, Trans. Amer. Math. Soc, to appear.

[8] K. Bringmann and K. Ono, Arithmetic properties of coefficients of half-integral weight Maass-Poincaré series, Math. Ann. 337 (2007), 591-612.

[9] K. Bringmann and K. Ono, Lifting cusp forms to Maass forms with an application to partitions, Proc. Nat. Acad. Sci., USA 104 (2007), 3725-3731.

[10] K. Bringmann and O. Richter, Zagier-type dualities and lifting maps for harmonic Maass-Jacobi forms, Adv. Math. 225 (2010), 2298-2315.

[11] J. Bruinier and J. Funke, On two geometric theta lifts, Duke Math. J. 125 (2004), 45-90.

[12] J. Bruinier and K. Ono, Arithmetic of Borcherds' exponents, Math. Ann. 327 (2003), $293-303$.

[13] J. Bruinier and K. Ono, Heegner divisors, L-functions, and harmonic weak Maass forms, Ann. of Math. 172 (2010), 2135-2181.

[14] J. Bruinier, K. Ono, and R. Rhoades, Differential Operators and Vanishing of Hecke Eigenvalues, Math. Ann. 342 (2008), 673-693. 
[15] Y. Choie, M. Knopp, and W. Pribitkin, Niebur Integrals, Mock Automorphic Forms and Harmonic Maass Forms: A Retrospective on the Work of the Rademacher School, preprint.

[16] W. Duke and P. Jenkins, On the zeros and coefficients of certain weakly holomorphic modular forms, Pure Appl. Math. Q. 4 (2008), 1327-1340.

[17] W. Duke, Ö. Imamoḡlu, and Â. Tóth, Cycle Integrals of the j-function and Mock Modular Forms, Ann. of Math., to appear.

[18] M. Eichler, Eine Verallgemeinerung der abelschen Integrale, Math. Z. 67 (1957), 267-298.

[19] J. Fay, Fourier coefficients of the resolvent for a Fuchsian group, J. reine und angew. Math. 293-294 (1977), 143-203.

[20] A. Folsom and K. Ono, Duality involving the mock theta function $f(q)$, J. Lond. Math. Soc. 77 (2008), $320-334$.

[21] K. Fricke, Analytische und p-adische Aspekte von klassischen und Mock-Modulformen, Ph.D. thesis, Max-Planck Insitute of Mathematics - Bonn, Germany, in preparation.

[22] P. Guerzhoy, Z. Kent, and K. Ono, p-adic coupling of mock modular forms and shadows, Proc. Nat. Acad. Sci. USA 107 (2010), 6169-6174.

[23] H. Iwaniec, Topics in classical automorphic forms, Graduate studies in Mathematics 53, Amer. Math. Soc., Providence, RI, USA, 1997.

[24] P. Jenkins, Kloosterman sums and traces of singular moduli, J. Number Theory 117 (2006), 301-314.

[25] M. Knopp, Construction of automorphic forms on H-groups and supplementary Fourier series, Trans. Amer. Math. Soc. 103 (1962), 168-188.

[26] M. Knopp, Modular functions in analytic number theory, Amer. Math. Soc., Chelsea Publishing, Providence, RI, 1993.

[27] M. Knopp, On the Fourier coefficients of cusp forms having small positive weight, Theta FunctionsBowdoin 1987, Part 2 (Brunswick, ME, 1987) eds. L. Ehrenpreis et al., Proc. Sympos. Pure Math. 49, Part 2, Amer. Math. Soc., Providence, RI, 1989, 111-127.

[28] M. Knopp and J. Lehner, On complementary automorphic forms and supplementary Fourier series, Illinois J. Math. 6 (1962) 98-106.

[29] N. Kuznetsov, The Petersson conjecture for cusp forms of weight zero and the Linnik conjecture. Sums of Kloosterman sums, Mat. Sb. (N.S.) 111(153) (1980), 334-383, 479 (in Russian); English translation: Math. USSR Sbornik 39 (1981), 299-342.

[30] H. Neunhöffer, Über die analytische Fortsetzung von Poincaréreihen, S.-B. Heidelberger Akad. Wiss. Math.-Natur Kl. 2 (1973), 33-90.

[31] W. Pribitkin, The Fourier coefficients of modular forms and Neibur modular integrals having small positive weight, I, Acta Arith. 91 (1999), 291-309.

[32] W. Pribitkin, The Fourier Coefficients of Modular Forms and Neibur Modular Integrals Having Small Positive Weight, II, Acta Arith. 93 (2000), 343-358.

[33] H. Rademacher, On the expansion of the partition function in a series, Ann. of Math. (2) 44 (1943), $416-422$.

[34] S. Ramanujan, The lost notebook and other unpublished papers, Narosa, New Delhi, 1988.

[35] J. Rouse, Zagier duality for the exponents of Borcherds products for Hilbert modular forms, J. Lond. Math. Soc. 73 (2006), 339-354.

[36] G. Watson, The final problem: an account of the mock theta functions, J. London Math. Soc. 11 (1936), $55-80$.

[37] G. Watson, The mock theta functions (2), Proc. London Math. Soc. 42 (1937), 274-304.

[38] E. Whittaker and G. Watson, A course in modern analysis, 4th ed., Cambridge University Press, Cambridge, England (1990), 620 pp.

[39] D. Zagier, Traces of Singular Moduli, Motives, polylogarithms and Hodge theory, Part I (Irvine, CA, 1998), 211-244, Int. Press Lect. Ser., 3, I, Int. Press, Somerville, MA, 2002.

[40] D. Zagier, Ramanujan's mock theta functions and their applications [d'après Zwegers and BringmannOno], Sém. Bourbaki, Astérisque 326 (2009), 143-164.

[41] S. Zwegers, Mock $\theta$-functions and Real Analytic Modular Forms. Contemp. Math. 291 (2001), 269-277. 
[42] S. Zwegers, Mock theta functions, Ph.D. Thesis, Universiteit Utrecht, 2002.

[43] S. Zwegers, The Folsom-Ono grid contains only integers, Proc. Amer. Math. Soc. 137 (2009), 1579-1584.

Mathematical Institute, University of Cologne, Weyertal 86-90, 50931 Cologne, GerMANY

E-mail address: kbringma@math.uni-koeln.de

Mathematical Institute, University of Cologne, Weyertal 86-90, 50931 Cologne, GerMANY

E-mail address: bkane@math.uni-koeln.de

Stanford University, Serra Mall Bldg. 380, Stanford, CA 94305

E-mail address: rrhoades@stanford.edu 